\title{
Management of Sulphone-resistant Lepromatous Leprosy
}

\author{
W. H. JOPLING \\ Formerly of The Hospital for Tropical Diseases, \\ St Pancras Way, London NW1, U.K.
}

The first thing to be said on the subject of dapsone resistance in leprosy is that we know very little about it apart from the fact that resistant strains of Mycobacterium leprae arise by spontaneous mutation, that resistance appears to be on the increase, and that up to the present time it has been reported only in lepromatous leprosy. I have never encountered it in tuberculoid or borderline leprosy, and I have not heard of anyone who has, although I accept that one day it may be reported as a rare occurrence in Mitsuda-negative borderline leprosy (i.e. $\mathrm{BL}$ and $\mathrm{BB})$.

When relapse occurs after a period of initial response to chemotherapy, and the patient has been taking small doses of dapsone, the clinician is tempted to assume that a dapsone-resistant strain of $M$. leprue has arisen because of small dosage, and when relapse occurs in a patient who has been on large doses, and it is known that the patient (like the majority of lepromatous patients) in unreliable on treatment, the clinician is tempted to blame irregular treatment, but, to the best of my knowledge, both these hypotheses are still without scientific proof. It should be noted that when a strain of pathogenic bacteria develops resistance to a given drug, the infection can sometimes be controlled by giving greatly increased dosage of that particular drug, and this probably accounts for the fact that a patient harbouring dapsone-resistant and dapsone-sensitive strains of $M$. leprae is less likely to relapse on large, rather than small, doses of dapsone, and vice versa. Therefore a case can be made for giving adult lepromatous patients $100 \mathrm{mg}$ dapsone daily even though much smaller doses are effective against dapsonesensitive strains. Readers will be familiar with the reports of Russell and colleagues from Papua New Guinea showing that intramuscular injections of acedapsone (DADDS; Hansolar) every $2 \frac{1}{2}$ months, liberating only $2.4 \mathrm{mg}$ dapsone daily, gave results comparable with those obtained from standard oral dapsone dosage of $100 \mathrm{mg}$ daily.

The therapeutic policy which I would advise in order to reduce the likelihood of relapse in lepromatous leprosy while under treatment is the one outlined by Waters and Helmy (1974), namely, to give adults $100 \mathrm{mg}$ dapsone daily from the outset, using steroid or thalidomide (without reduction of dapsone dosage) should serious Type 2 lepra reaction (ENL reaction) occur. It is my policy, if relapse does occur, to add clofazimine (Lamprene; B663) to treatment in a dosage of $100 \mathrm{mg}$ twice a week, and results have been consistently satisfactory; pigmentary changes, 
even in light-skinned patients, have been absent. It is important that dapsone should be continued, together with clofazimine, so that it can continue to inhibit the dapsone-sensitive strains, thus leaving clofazimine to inhibit the dapsoneresistant strains. I am opposed to the policy (which is widely being proposed) of giving combined treatment routinely and de novo in lepromatous leprosy before the problem of overcoming the high defaulter rate among outpatients has been tackled, for patients who default on 1 drug are more likely to default on 2 , and combined treatment will then have the disadvantage of being expensive as well as wasteful. I would prefer to see dapsone given alone, combined with an all-out effort to overcome defaulting, with the proviso that skin smears are taken routinely every 6 months in order to get early intimation of relapse (long before clinical relapse can be observed); 2 smears should include the dorsa of fingers (Ridley et al., 1976), preferably over the first phalanges. Only if there is an increase in solid-staining bacilli need action be taken, and this consists in assessing bacteriological response to regular dosage of dapsone by intramuscular injection over a period of 3 months; testing in the mouse foot-pad calls for a specialized laboratory and, in any case, takes more than twice as long to prove. I would like to sound a note of warning against assuming that a patient who relapses is harbouring dapsone-resistant bacilli, for it is equally possible that he has not been taking the drug, and therefore, dapsone resistance must be proved before being accepted.

The above remarks apply to the management of lepromatous leprosy, as it is in this type that the problem of dapsone resistance is important, therefore observations on the treatment of borderline leprosy are irrelevant at this stage, but I would like to stress that large doses of dapsone in this type of the disease are both unnecessary and dangerous (Jopling, 1977).

\section{References}

Jopling, W. H. (1977). Letter in Lancet $i, 44$.

Ridley, M., Jopling, W. H. and Ridley, D. S. (1976). Acid-fast bacilli in the fingers of long-treated lepromatous patients. Lepr. Rev. 47, 93.

Waters, M. F. R. and Helmy, H. S. (1974). The relationship of dapsone (DDS) therapy to erythema nodosum leprosum. Is it direct or indirect? Lepr. Rev. 45, 299. 